\title{
Fast and pervasive heat transport induced by multiple locked modes in DIII-D
}

\author{
Q. $\mathrm{Hu}^{1}, \mathrm{X} . \mathrm{Du}^{2}, \mathrm{Q} . \mathrm{Yu}^{3}$, N.C. $\operatorname{Logan}^{1}, \mathrm{E} . \mathrm{Kolemen}^{4}, \mathrm{R}$. \\ Nazikian $^{1}$, and Z.H. Jiang ${ }^{5}$ \\ ${ }^{1}$ Princeton Plasma Physics Laboratory, Princeton NJ 08543-0451, USA \\ ${ }^{2}$ General Atomics, PO Box 85608 San Diego, CA 92186-5608, USA \\ ${ }^{3}$ Max-Plank-Institut für Plasmaphysik, 85748 Garching, Germany \\ ${ }^{4}$ Mechanical and Aerospace Engineering, Princeton University, Princeton NJ, USA \\ ${ }^{5}$ International Joint Research Laboratory of Magnetic Confinement Fusion and \\ Plasma Physics, State Key Laboratory of Advanced Electromagnetic Engineering and \\ Technology, School of Electrical and Electronic Engineering, Huazhong University of \\ Science and Technology, Wuhan, 430074, China
}

E-mail: qhu@pppl.gov

\begin{abstract}
This work presents the impact that multiple island chains co-existent across a tokamak plasma profile have on the heat transport and final temperature of that plasma. Numerical studies using the TM1 code show that error fields (EFs) with multiple poloidal components accelerate the core field penetration compared to pure $m / n=2 / 1$ EF penetration (here $m$ and $n$ are the poloidal and toroidal mode numbers respectively). After field penetration, locked magnetic islands of $m / n=2 / 1,3 / 1$ and $4 / 1$ flatten the temperature at the corresponding rational surfaces. The co-existence of these islands significantly enhances the plasma heat transport throughout a wide swath of plasma from the core $2 / 1$ rational surface to plasma edge. The electron temperature $T_{e}$ profile from $2 / 1$ to $4 / 1$ rational surfaces can be nearly flattened even if there is no island overlap, and the temperature inside each island is determined by the boundary temperature at the outboard separatrix of the island. The resulting central $T_{e}$ decreases by more than $50 \%$, in good agreement with experimental observations and much lower than modeling with only a single $2 / 1$ locked island. Further comparisons of the $T_{e}$ profile between numerical modeling and DIII-D experiment indicates that the observed reduction in the edge temperature requires edge island overlap and stochasticity. Numerical scans reveal the $T_{e}$ profile decreases further when large EF amplitudes create larger islands, wider edge stochastic regions and secondary island structures. Scans of the relative phase between EF harmonics reveal that the $3 / 1$ island width is most sensitive to the island phase and the central $T_{e}$ changes with the $3 / 1$ island width. These results indicate that the coexistence of multiple LMs in tokamak plasmas deteriorate thermal confinement more than the sum of their isolated impacts would and that this may be responsible for the fast thermal quench observed prior to major disruptions.
\end{abstract}

Keywords: multiple locked modes, heat transport, disruption, thermal quench, error field 


\section{Introduction}

Plasma disruptions seriously challenge the safe operation of tokamaks because of the possible damage caused by large thermal loads on invessel components and strong electromagnetic forces on surrounding conductors [1-3]. Studies in JET [4, 5], DIII-D [6] and NSTX [7] find that locked modes (LMs) are one of the main physical causes of disruptions in current devices. These are tearing modes (TMs) locked to the intrinsic error field (EF) and resistive wall [8-10]. TMs can be naturally unstable or driven by magnetic perturbations from EFs and externally applied resonant magnetic perturbations (RMPs) [9-11]. These LMs form locked magnetic island chains at the corresponding rational surface and cause substantially deterioration of energy confinement [13].

Extensive studies have been devoted to understand the influence of LMs or magnetic islands on the thermal quench (TQ) during disruptions [12-19]. Multiple rotating modes are observed to correlate with TQs on many tokamak devices. In the density limit discharges of JET [12] and ASDEX [13], rotating precursor modes with different poloidal mode numbers are observed to cause several minor disruptions (with only thermal collapses) before ultimately leading to a TQ and major disruption. Rotating $m / n=2 / 1,3 / 2$ and $5 / 3(m$ and $n$ are the poloidal and toroidal mode numbers) modes are observed to coexist preceding major disruptions on TOSCA [14], and TQs happen only when the amplitudes of rotating $2 / 1$ and $3 / 2$ modes exceed a threshold. On KSTAR, two types of fast thermal collapses correlated with $2 / 1$ or $1 / 1$ locked magnetic islands are observed by using quasi-3D electron cyclotron emission imaging (ECEI) system [17]. On DIII-D, the coexistence of multiple LMs is derived from magnetic measurements that indicate the growth and overlap of these multiple LMs leads to thermal collapses [18]. In recent DIII-D experiments using a dual tangential soft X-ray imaging (DSXI) system, the topology of locked magnetic is- land chains with multiple helicities $(\mathrm{m} / \mathrm{n}=$ $2 / 1,3 / 1$ and 4/1) governs the cooling process in the plasma peripheral region [19]. All these observations reveal that multiple magnetic island chains play an essential role in the TQ process.

Multiple LMs usually happen after the occurence of mode locking or field penetration $[18,19]$, due to the braking and destabilizing effects caused by the wide bandwidth of EF $[11,20,21]$ and toroidal coupling effects [2224]. These multiple LMs challenge the study of the transition process from LMs to TQ in both experiment and theory. In the experiment, it is difficult to detect and distinguish multiple locked island chains due to loss of mode rotation [18]. In theoretical modeling, plasma transport and nonlinear effects couple the multiple LMs. As a result, the transition process from mode locking to TQ has been poorly understood, though there have been many investigations since the 1970's [2530]. Most notably, the TQ is found to occur when the amplitude of LMs reaches a distinct level on JET, ASDEX Upgrade and COMPASS [31]. The information of LMs is seen as precursors to disruption and can be used to determine thresholds for simple disruption prediction schemes. Therefore, improving the physical understanding of the transition process from LMs to TQ might lead to better disruption prediction and avoidance, which is essential for the success of ITER [2, 3].

Motivated by the direct observation of multiple LMs on DIII-D [19], the effect of multiple LMs on heat transport is presented in this paper based on nonlinear magnetohydrodynamic (MHD) modeling. In section 2, a typical low density EF penetration discharge observed on DIII-D is introduced. The evolution of the electron temperature $\left(T_{e}\right)$ profile shows deteriorating energy confinement followed by a sharp TQ. To understand how the multiple LMs affect heat transport, a nonlinear theoretical model based on reduced MHD equations is utilized to simulate EFs penetration and the associated evolution of the $T_{e}$ profile in section 3. The simulations are initialized with 
the DIII-D equilibrium prior to EF penetration. Compared to a single $2 / 1 \mathrm{LM}$, it is found that multiple helicity LMs further decrease $T_{e}$. The detailed evolution of $T_{e}$ shows that the $4 / 1$ locked island sets the outer boundary $T_{e}$ for the $3 / 1$ locked island, and the $3 / 1 \mathrm{LM}$ sets that for the $2 / 1$, resulting in a global degradation of $T_{e}$ from the edge to the core. Scanning the modeled EF amplitude reveals that island overlap between the $3 / 1$ and the $4 / 1$ is easily obtained, causing stochastic fields at plasma edge. The modeled $T_{e}$ profile evolution with the presence of edge stochasticity is qualitatively consistent with experimental profiles. Stronger EF amplitudes also lead to larger island width, secondary island structures, wider stochastic regions and lower $T_{e}$. Finally, the phase of $2 / 1,3 / 1$ and $4 / 1$ EF is scanned separately to study the influence of multiple LMs on $T_{e}$ with different alignment phase. The varying central $T_{e}$ is found to correlate with $3 / 1$ island width. The concluding discussion and summary are given in section 4 .

\section{Coexisting of multiple LMs after EFs penetration}

EFs penetration triggers a specific class of LMs that typically appear in plasmas with low density [32-34]. These specific LMs, are born locked (i.e. there is no prior phase when the mode rotates with the plasma). An ohmic $q_{95} \sim 4$ plasma with these LMs evolving to disruption is shown in figure 1. This DIII$\mathrm{D}$ experiment is performed with inner wall limited, oval plasma shaping. The intrinsic $n=1 \mathrm{EF}$ is known to be dominantly due to a bus-work locally feeding the current to the toroidal field $\left(B_{T}\right)$ coil and from shifts and tilts of outer poloidal field $(\mathrm{F})$ coils that make the plasma equilibrium $[33,35]$. In the experiment, the central line-averaged electron density is about $1.6 \times 10^{19} \mathrm{~m}^{-3}$ and keeps constant before EF penetration (figure 1(e)). The amplitude of the $2 / 1,3 / 1$ and $4 / 1 \mathrm{EFs}$, at the corresponding rational surfaces, are calculated to be about $2 \mathrm{G}, 1.2 \mathrm{G}$ and $2.1 \mathrm{G}$ with the phase of $\Phi=210^{\circ}, 80^{\circ}$ and $270^{\circ}$ (in left-hand coordinate) by the SURFMN vacuum code [36].
Electron cyclotron heating $(\mathrm{ECH})$ is turned off at $5 \mathrm{~s}$ (figure 1(b)), and EF penetration happens at $5.05 \mathrm{~s}$ as indicated by the fast growth of the $n=1$ poloidal magnetic perturbation $B_{p}^{n=1}$ shown in figure 1(c). Similar to the phase III in Ref [34], after EF penetration, the width of locked island increases with the increasing $B_{p}$ in the time interval of 5.05 $\mathrm{s}<t<5.24 \mathrm{~s}$, according to the relationship between magnetic island width and poloidal magnetic perturbation that $W \propto B_{p}^{0.5}[10]$. The increasing locked island decreases $T_{e}$ from edge to central plasma as shown in figure $1(d)$. After a period of saturation, the amplitude of $B_{p}^{n=1}$ begins to increase rapidly. This large $n=1$ field leads to a TQ and major disruption at $5.446 \mathrm{~s}$, corresponding to phase IV in Ref. [34].

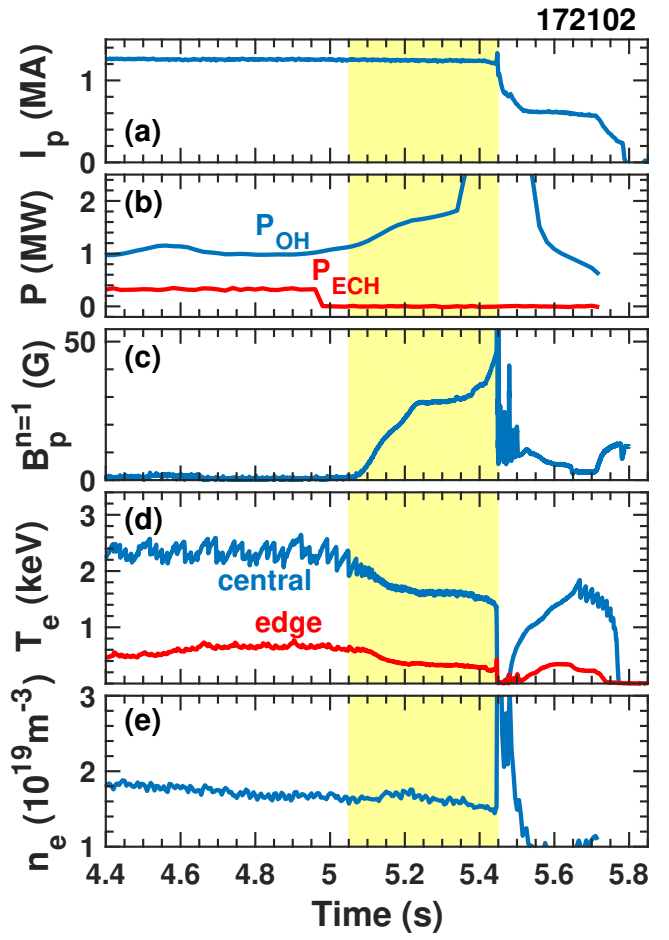

Figure 1. The plasma evolution during EF penetration in the low density Ohmic discharge 172102. Time evolution of (a) plasma current $I_{p}$, (b) Ohmic heating power $P_{O H}$ and ECH heating power $P_{E C H}$, (c) $n=1$ poloidal magnetic perturbation $B_{p}^{n=1}$, (d) $T_{e}$ at plasma core and edge $T_{e}$ measured by electron cyclotron emission (ECE) and (e) core electron density $n_{e}$. The shadowed region indicates the transition process from multiple LMs to TQ.

The Thomson scattering (TS) and Elec- 
tron Cyclotron Emission (ECE) diagnostics in DIII-D are used together to measure the $T_{e}$ profile [37]. Figure 2 presents TS measurements of $T_{e}$ profile at different times during EF penetration phase. A flattening is noticeable in the $T_{e}$ profile at the $q=2,3$ and 4 locations, indicating the formation of $2 / 1,3 / 1$ and $4 / 1$ magnetic islands at the corresponding rational surfaces. These modes have been proven to be locked magnetic islands and not kink-like modes in Ref. [19] using DSXI measurements. As time evolves, the flattened regions become wider and the $T_{e}$ in each region decreases due to the growth of island widths. The growing magnetic islands get closer to neighboring ones as indicated by the decreasing boundary distance between the flattening regions. The flattening regions due to $3 / 1$ and $4 / 1$ islands almost overlap prior to the TQ. After the TQ, the $T_{e}$ profile at $5.48 \mathrm{~s}$ is almost flattened from $q=2$ to plasma edge.

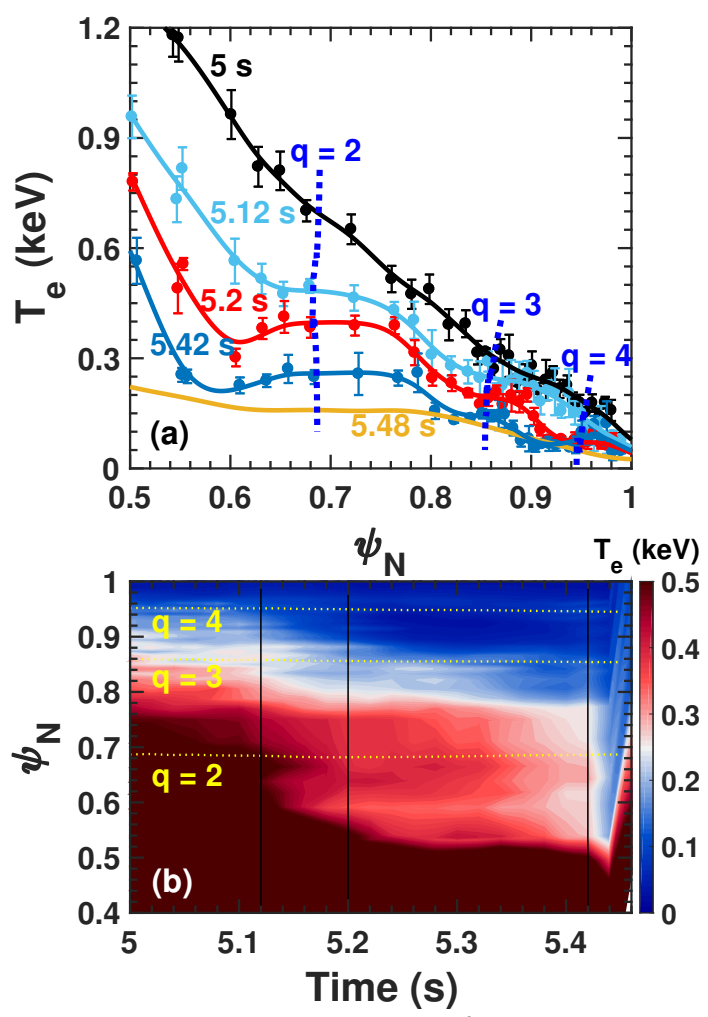

Figure 2. Detailed evolutions of the electron temperature in shot 172102. In (a), profiles of $T_{e}$ are shown at $5 \mathrm{~s}, 5.12 \mathrm{~s}, 5.2 \mathrm{~s}, 5.42 \mathrm{~s}$ and $5.48 \mathrm{~s}$ with the position of the $q=2,3$ and 4 rational surfaces designated by blue dotted curves. In (b), the evolution of $T_{e}$ profile is shown, here the vertical solid lines indicate the time slices shown in (a).
The evolution of $T_{e}$ profiles in figure 2 is unique to the type of transport caused by multiple LMs. The global $T_{e}$ is substantially decreased, and the stair like $T_{e}$ profile in figure 2(a) differs from that caused by a single island [38]. It is well understood in both theory $[29,30]$ and experiment $[38,39]$ that, in the presence of a single island, $T_{e}$ is flattened across the island due to the extreme anisotropic heat transport. However, there are few studies on the presence of multiple island chains with different helicity. In section 3 of this paper we will take a more in depth study on the nonlinear physics of this heat transport in the presence of multiple island chains.

\section{Numerical results}

In order to study the effect of multiple LMs on heat transport addressed in Section 2, a theoretical model is introduced in Subsection 3.1 and numerical results modeling the dishcarge in question are presented in Subsections 3.2, 3.3 and 3.4 .

\subsection{Theoretical model}

The model uses a straight cylindrical, circular cross section tokamak for simplicity when calculating the nonlinear evolution of MHD and transport from multiple magnetic islands. The magnetic field is defined as $\boldsymbol{B}=B_{t} \mathbf{e}_{t}+\nabla \psi \times \mathbf{e}_{t}$, where $\psi$ is the magnetic flux function. The plasma velocity is defined as $v=\nabla \phi \times \mathbf{e}_{t}$, where $\phi$ is the stream function. The basic equations utilized here are Ohm's law, the equation of motion (after taking the operator $\left.\mathbf{e}_{t} \cdot \nabla \times\right)$ and the energy conservation equation. Normalizing all the lengths to the minor radius $a$, the time $t$ to the resistive time $\tau_{R}=a^{2} \mu_{0} / \eta$, the helical flux $\psi$ to $a B_{t}$, the velocity $v$ to $a / \tau_{R}$, and the electron temperature $T_{e}$ to its value at the magnetic axis, these equations become,

$$
\begin{aligned}
& \frac{d \psi}{d t} \quad=E-\eta j \\
& \frac{d U}{d t} \quad=-S^{2} \nabla_{\|} j+\mu \nabla_{\perp}^{2} U+S_{m} \\
& \frac{3}{2} n_{e} \frac{d T_{e}}{d t}=n_{e} \nabla_{\|}\left(\chi_{\|} \nabla_{\|} T_{e}\right)
\end{aligned}
$$




$$
+n_{e} \nabla_{\perp}\left(\chi_{\perp} \nabla_{\perp} T_{e}\right)+S_{p}
$$

where $d / d t=\partial / \partial t+v_{\perp} \cdot \nabla$. Plasma current density $j$ is derived according to $\boldsymbol{j}=\nabla \times \boldsymbol{B}$. $n_{e}$ is the electron density, $\eta$ is the normalized plasma resistivity and $E$ is the equilibrium electric field. The magnetic Reynolds number $S=\tau_{R} / \tau_{A}$, where $\tau_{A}=a / V_{A}$ is the toroidal Alfvèn time. $U=\nabla_{\perp}^{2} \phi$ is the plasma vorticity, and $\mu$ is the plasma viscosity. $\chi_{\|}$and $\chi_{\perp}$ are the parallel and perpendicular heat conductivities, $S_{p}$ is the heating power and $S_{m}$ in equation (2) is the momentum source which leads to an equilibrium plasma rotation. Here, $\eta, S, S_{m}$ and $S_{p}$ are temporal constants, and $E, \mu, n_{e}, \chi_{\|}$and $\chi_{\perp}$ are spatial constants.

Equations (1)-(3) provide a reduced MHD model for modeling EF penetration and nonlinear growth of LMs. It should be mentioned that the diamagnetic drift has not been included in our model utilized here, which is important in determining the TM stability for high $\beta$ plasmas with large electron pressure gradient. Equations (1)-(3) are solved simultaneously using the initial value code TM1 [40], which has been used for modeling the nonlinear growth and saturation of NTMs and their stabilization by radio frequency $(\mathrm{RF})$ current $[41,42]$ as well as the effect of resonant magnetic perturbations (RMPs) on resistive TMs [43, 44]. Dedicated numerical methods are utilized in the code to keep the numerical error at a very low level even for high values of $S$ and $\chi_{\|} / \chi_{\perp}[41,45,46]$.

The calculations in this work only include multiple resonant helicity perturbations with $m / n=2 / 1,3 / 1$ and $4 / 1$ (non-resonant components are not included). In addition to the fundamental harmonic, higher harmonic perturbations (including from $2^{\text {nd }}$ to $7^{\text {th }}$ harmonics for each helicity) as well as the change in the equilibrium quantities (the $m / n=0 / 0$ component) are self-consistently calculated. The toroidal magnetic field is taken to be a constant and the toroidal mode coupling is neglected. Fourier decomposition in the poloidal and toroidal directions and finite differences along the radial direction are utilized in the code. The calculation region is from the magnetic axis at $r=0$ to the plasma edge at $r=a$. The boundary conditions are as the following [47].

(a) The radial gradients of all quantities are zero at $r=0$.

(b) All the perturbations $(m / n \neq 0 / 0)$ are zero at $r=a$ except for the $m / n=2 / 1$, $3 / 1$ and 4/1 magnetic perturbation given by the following equation (4) to take into account the EF.

(c) All the equilibrium $(m / n=0 / 0)$ quantities take the same value as the original equilibrium ones at $r=a$.

The effect of the EF is taken into account by the boundary condition

$\psi_{m / n}(r=a)=\psi_{a} a B_{t} \cos \left(m \theta+n \varphi+\Phi_{0}\right)$,

where $\psi_{a}$ and $\Phi$ describe the amplitude and phase of the applied EF of the $m / n$ component at $r=a$. The radial $\mathrm{EF}$ at $r=a$ is given by $b_{r a}=m \psi_{a} B_{t}$. The driving $\mathrm{EF}$ is taken from and kept at the vacuum RMP value at the plasma boundary for each harmonic.

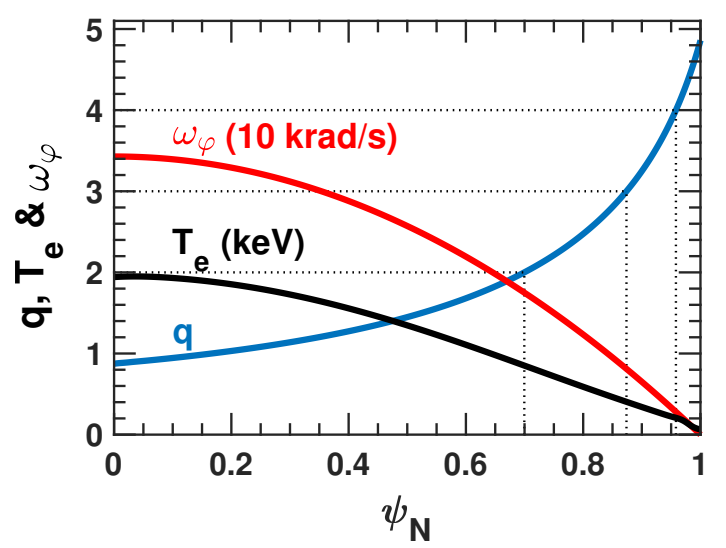

Figure 3. These quilibrium profiles of safety factor, $T_{e}$ and toroidal angular rotation taken from 5 $\mathrm{s}$ into the shot shown in figure 1 are used for modeling. The $q=2,3$ and 4 rational surfaces locate at $\psi_{N}=0.7,0.87$ and 0.96 , respectively.

The input parameters are based on the experimental parameters and equilibrium at 5 s of shot 172102 as shown in figure 3 . The toroidal magnetic field on axis is $-1.92 \mathrm{~T}$ and the plasma minor and major radii are $a=0.63$ $\mathrm{m}$ and $R=1.7 \mathrm{~m}$. The $q=2,3$ and 4 rational surfaces are located at $\psi_{N}=0.7,0.87$ and 0.96 , respectively. These parameters lead 
to the normalized parameters $S=5 \times 10^{7}$, $\chi_{\|}=2.82 \times 10^{9}\left(a^{2} / \tau_{R}\right)$. Here, $\chi_{\|}=\nu_{T e} / k_{\|}$is used for calculating $\chi_{\|}$and $\nu_{T e}=\left(T_{e} / m_{e}\right)^{1 / 2}$ is the electron thermal velocity [48]. It should be noted that the precise form of $\chi_{\|}$for a high temperature plasma is more complex as shown in Ref [48]. Assuming the perpendicular heat diffusivity and the plasma viscosity to be at the anomalous transport level of $0.5 \mathrm{~m}^{2} / \mathrm{s}$, in the normalized units they are $\chi_{\perp}=\mu=2.8\left(a^{2} / \tau_{R}\right)$, and the ratio between $\chi_{\|}$and $\chi_{\perp}$ is $\chi_{\|} / \chi_{\perp} \sim 10^{9}$. These parameters are the input for our calculations except mentioned elsewhere.

The angular rotation $\omega$ shown in figure 3 is derived by toroidal plasma rotation according to $\omega=V_{\Phi} / R$, and the perpendicular rotation $v=\omega r$ is utilized in our model to simulate the plasma rotation. As we know, in tokamak experiments due to neoclassical effects the plasma rotation is mostly in the toroidal direction. While in our model, the reduction of the poloidal rotation is caused by the poloidal electromagnetic force. This leads to two modifications $[8,9]$ : (a) the electromagnetic force to slow down the rotation in the toroidal direction is smaller by a factor $(n / m)\left(r_{s} / R\right)$ compared with that in the poloidal direction. (b) To have the same mode frequency due to the plasma rotation, the toroidal rotation speed should be $(m / n)\left(R / r_{s}\right)$ times larger than the poloidal one. These two effects lead to a larger ratio of the viscous force to the electromagnetic force, by a factor $(m / n)^{2}\left(R / r_{s}\right)^{2}$ for the toroidal rotation case. As a result, the penetration threshold for toroidal rotation case will be modified by a factor of $(m / n)^{2}\left(R / r_{s}\right)^{2}$ compared to poloidal rotation case for quantitative studies [42].

It should be noted that the redistribution of the current density profile due to changes in $T_{e}$ and hence resistivity has not been taken into account in the modeling, though the locations of $q=2,3$ and 4 rational surfaces change little until disruption as shown in figure 2. As a result, the time scale of numerical evolution is not consistent with experiment. However, the qualitative understanding of the influence of multiple LMs on heat transport is still valid. Therefore, the following numerical results will focus on the qualitative understanding and comparison with experiment.

\subsection{Heat transport with single helicity EF penetration}

Before presenting the results of multiple LMs, it is useful to first study the effect of a single helicity locked island, to form a clear picture of EF penetration and the associated heat transport. In this subsection, results of single helicity EFs of $m / n=2 / 1,3 / 1$ and $4 / 1$ penetration are presented separately.

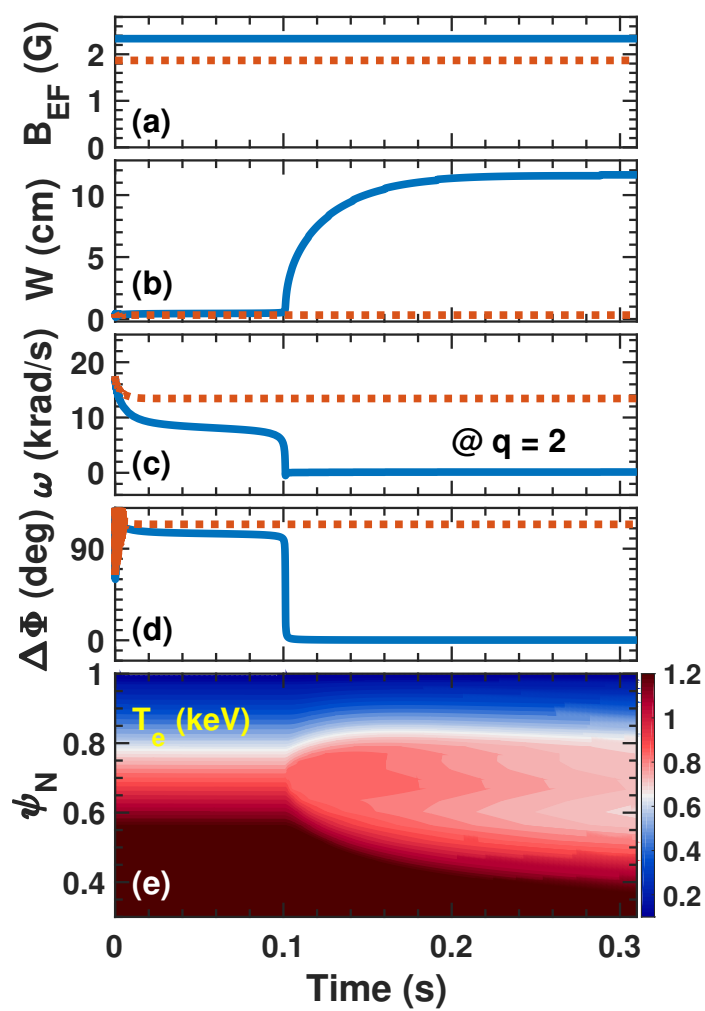

Figure 4. $m / n=2 / 1 \mathrm{EF}$ penetration case. Time evolution of (a) $2 / 1 \mathrm{EF}$ amplitude $B_{E F}$ at the $q$ $=2$ surface, (b) $2 / 1$ island width $W$, (c) angular rotation frequency $\omega$ at the $q=2$ surface, (d) phase difference $\Delta \Phi=\Phi-\Phi_{0}$ between the plasma response field $(\Phi)$ and vacuum field $\left(\Phi_{0}\right)$ for two cases with different 2/1 EF amplitude, $1.9 \mathrm{G}$ (red dotted curve) and $2.3 \mathrm{G}$ (blue curve). Time evolution of radial profile of $T_{e}$ for the penetrated case is shown in (e).

In figure 4 , the nonlinear evolution of $m / n=2 / 1$ quantities driven by a pure $2 / 1 \mathrm{EF}$ are shown for different EF amplitudes of $1.9 \mathrm{G}$ 
(red dotted curve) and $2.3 \mathrm{G}$ (blue curve). The 2/1 EF penetration threshold in this plasma is $2.1 \mathrm{G}$. In both cases, the simulation start time corresponds to $5 \mathrm{~s}$ in the experiment. For $B_{E F}=2.3 \mathrm{G}$, the evolution process can be divided into 2 stages: pre-penetration $(t<0.1$ $\mathrm{s})$ and post-penetration $(t>0.1 \mathrm{~s})$. In the pre-penetration stage, the plasma rotation at $q=2$ is slowed down gradually (figure 4(c)) by the resonant electromagnetic (EM) torque between the mode and the EF [10, 49]. The phase difference between the plasma response and the vacuum $\mathrm{EF}$ is about $110^{\circ}$ and decreases slightly, indicating a sheilding (kink) response in this stage [43]. At the end of this stage, the phase difference approaches $90^{\circ}$ and leads to stronger decelerating EM torque. This causes a much faster drop in both plasma rotation and phase difference, resulting in bifurcation from screening (kink) to field penetration $[10,44]$. In the post-penetration stage, the $2 / 1$ locked magnetic island begins to grow quickly and saturates at the width of $11.5 \mathrm{~cm}$ at $t=0.2 \mathrm{~s}$ (figure 4(e)). Associated with the growth of $2 / 1$ island, the $T_{e}$ is also decreased as shown in figure $4(\mathrm{e})$ and evolves with similar time scale of island growth. For EF with amplitudes lower than the penetration threshold, the EF is screened by the plasma with phase differences larger than $90^{\circ}$ and slight decreases in plasma rotation. In the $1.9 \mathrm{G}$ $\mathrm{EF}$ case for example, the rotation is decreased $30 \%$ and phase difference is about $110^{\circ}$.

In the pre-penetration stage of the $2.3 \mathrm{G}$ case, the EM torque due to the EF decreases the rotation at $q=2$ rational surface and also slows down the global plasma rotation because of plasma viscosity (momentum transport) as shown in figure 5(a). The rotation profile at $t=0.11 \mathrm{~s}$ indicates that the global rotation is decreased substantially with more than a $50 \%$ reduction across most of the profile. The global rotation deceleration decreases the EF penetration threshold of the other rational surfaces, as will be seen when modeling multiple helicities simultaneously. In the postpenetration stage, $T_{e}$ is flattened in the island region associated with the $2 / 1$ island. The $T_{e}$ is decreased core to the island. In contrast, this exhausted heat flux increases $T_{e}$ from the outside of the $2 / 1$ island region to the plasma edge (figure 5(b)). This discrepant change in $T_{e}$ on either side of a single LM is usually observed in minor disruptions $[17,18]$. At saturation, the central $T_{e}$ has decreased by about $20 \%$ and the edge $T_{e}$ returns to the initial profile since there is no additional exhaust heat flux from the inner region.

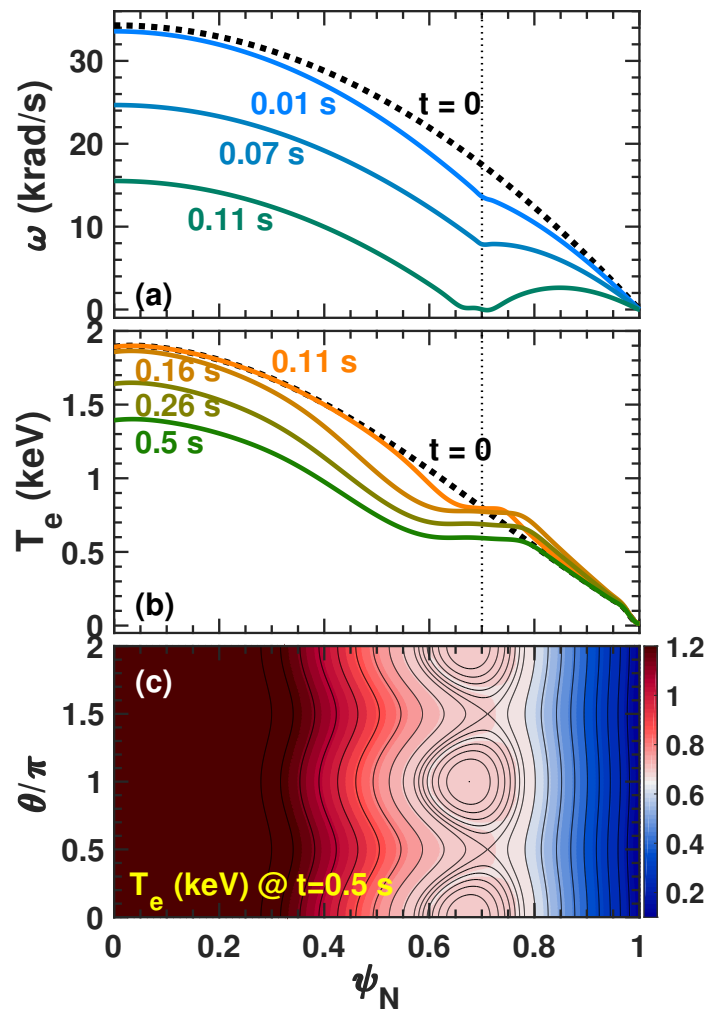

Figure 5. Radial profile of $m / n=0 / 0$ component (a) $\omega$ at $0,0.01,0.07$, and 0.11 seconds, and (b) $T_{e}$ at $0,0.11,0.16,0.26$, and 0.5 seconds. (c) 2-D profile of $T_{e}$ (including $0 / 0$ and harmonic components) and modeled helical flux surface at $0.4 \mathrm{~s}$ are shown for the $2.3 \mathrm{G}$ case from figure 4 .

Figure $5(\mathrm{c})$ shows the 2-D $T_{e}$ profile together with the 2/1 locked island topology at a single toroidal angle of 0 degrees. The radial profile of $T_{e}$ differs at the $\mathrm{O}$ and $\mathrm{X}$ points due to different local radial widths of the island. The large magnetic island also distorts the flux surfaces (kink response) outside of the island, in the next subsection results will show that this distortion of flux surface would affect the saturated island widths of any LMs at other rational surfaces. 

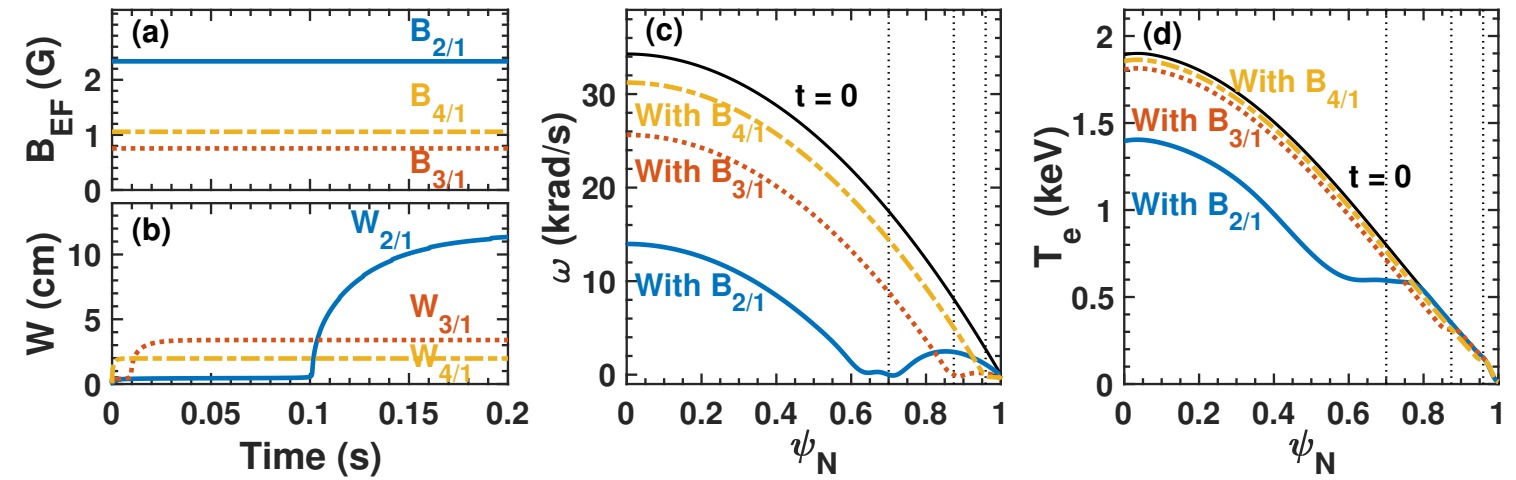

Figure 6. Comparison of pure $m / n=2 / 1,3 / 1$ and $4 / 1$ EF penetration. Time evolution of (a) EF amplitude $B_{E F}$, (b) island width $W$ for $m / n=2 / 1$ (blue curve), 3/1 (red dotted curve) and 4/1 (green dashed curve). Radial profiles of $m / n=0 / 0$ component (c) $\omega$ and (d) $T_{e}$ after locked island saturation.

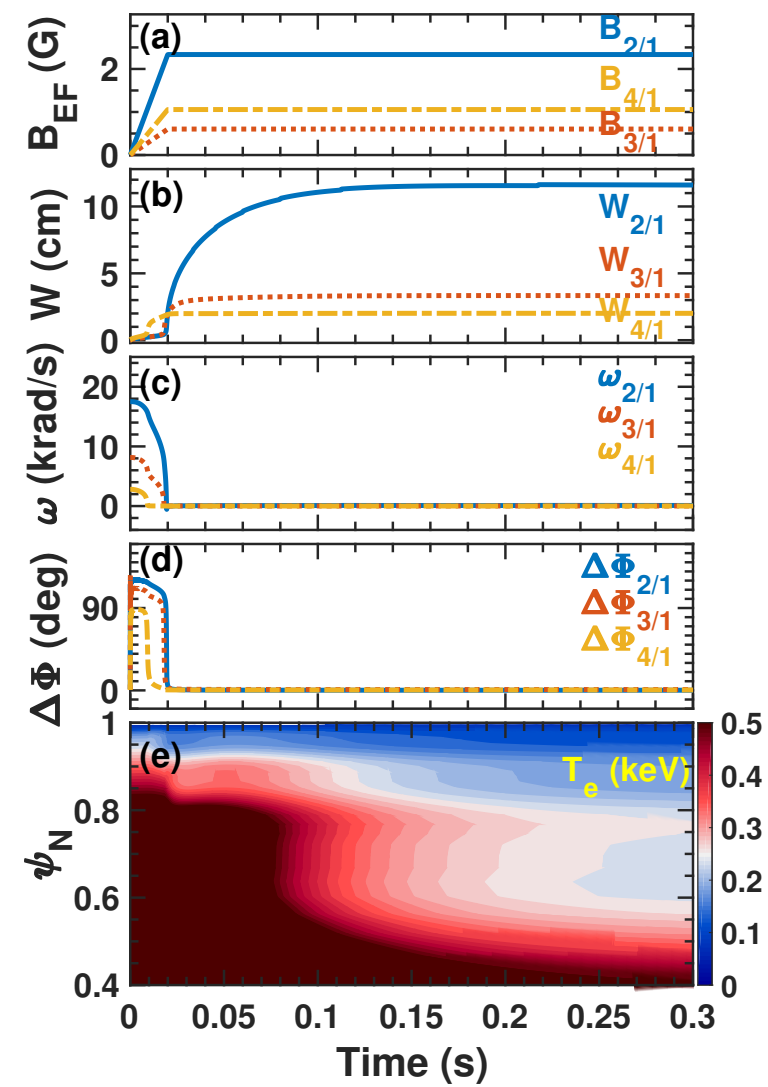

Figure 7. EF penetration with $m / n=2 / 1,3 / 1$ and $4 / 1$ components together. Time evolution of (a) EF amplitude $B_{E F}$ at corresponding rational surface, (b) island width $W$, (c) $\omega$, (d) phase difference $\Delta \Phi$ for $m / n=2 / 1,3 / 1$ and $4 / 1$. (e) Time evolution of radial profile of $T_{e}$.

In figure 6 , results of single $3 / 1$ (red curve) and 4/1 (yellow curve) EF penetration are shown alongside the previously discussed $2 / 1 \mathrm{EF}$ penetration for comparison. A 3/1 EF with an amplitude of $0.8 \mathrm{G}$ leads to field penetration and the corresponding locked island saturates at a width of $3.5 \mathrm{~cm}$. A 4/1
EF with an amplitude of $1.05 \mathrm{G}$ leads to a saturated $4 / 1$ island of $2 \mathrm{~cm}$. Similar to the $2 / 1 \mathrm{EF}$ penetration, the $3 / 1$ and $4 / 1 \mathrm{EFs}$ also slow down plasma rotation globally with near zero edge rotation as shown in figure $6(\mathrm{c})$. The smaller $3 / 1$ and 4/1 locked islands also cause weaker flattening of the $T_{e}$ profile, with $5 \%$ and $2 \%$ reduction in central $T_{e}$, respectively.

\subsection{Heat transport with multiple}

\section{LMs}

In this section, 2/1, 3/1 and 4/1 EFs are applied together to investigate the influence of multiple LMs on heat transport and compared with experiment.

In figure $7, m / n=2 / 1,3 / 1$ and $4 / 1$ EFs are applied together with the same amplitudes as in figure 6 (i.e. just above the penetration threshold for each helicity). In the simulation, the EFs are ramped up in $20 \mathrm{~ms}$ to ensure a clear evolution of the penetration process. The 4/1 EF penetrates first, followed by $3 / 1$ penetration and finally $2 / 1 \mathrm{EF}$ penetration. The final $2 / 1$ penetration occurs much earlier than the single helicity $2 / 1 \mathrm{EF}$ penetration $(t$ $=0.1 \mathrm{~s})$ in figure 4 , indicating the multiple helicity EF accelerates the processes leading to penetration. After the EF penetration of all the $2 / 1,3 / 1$ and $4 / 1$ helicities, the LMs saturate with widths similar to their respective single helicity cases and the plasma rotation is reduced to near zero at the $q=2,3$ and 4 rational surfaces. The evolution of $T_{e}$ profile in figure $7(\mathrm{e})$ is qualitatively similar to the experimental observations in figure 2(b). 

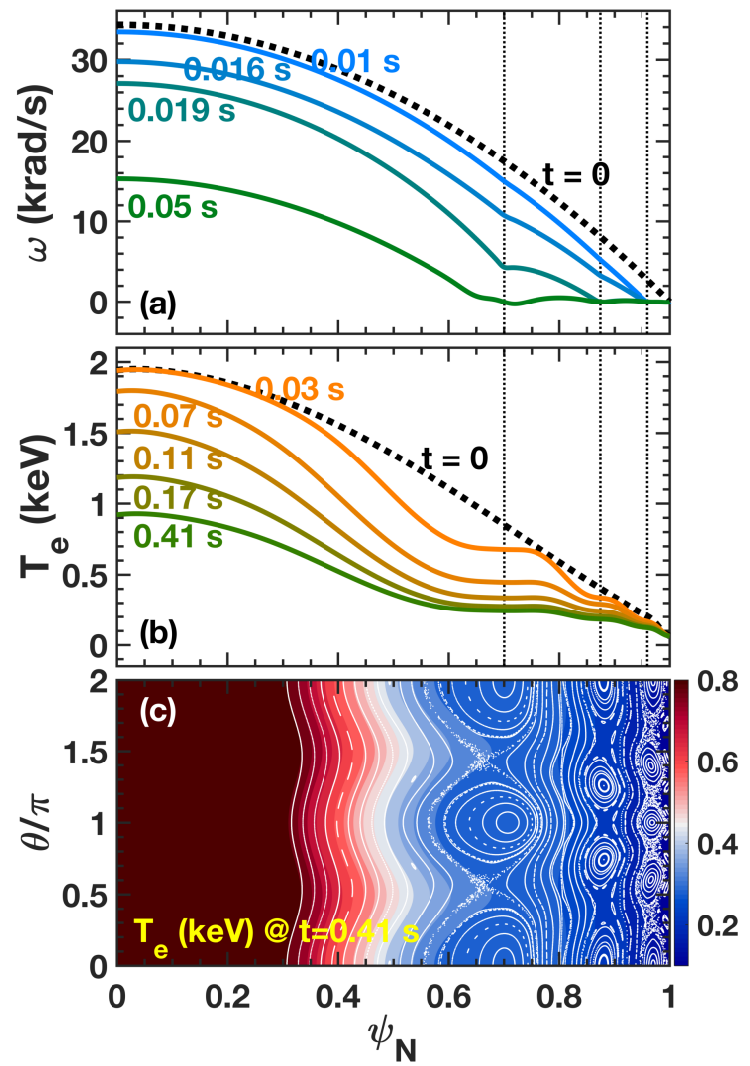

Figure 8. Corresponding to figure 7 , radial profile of $0 / 0$ component (a) $\omega$ at $t=0,0.01 \mathrm{~s}, 0.016$ $\mathrm{s}, 0.019 \mathrm{~s}, 0.05 \mathrm{~s}$, and (b) $T_{e}$ at $t=0,0.03 \mathrm{~s}, 0.07$ $\mathrm{s}, 0.11 \mathrm{~s}, 0.17 \mathrm{~s}, 0.41 \mathrm{~s}$. (c) 2 -D profile of $T_{e}$ and Poincaré plot of the flux surfaces (white) at $t=$ $0.41 \mathrm{~s}$. Here, field line tracing based on modeled magnetic response is used to represent the flux surfaces.

The detailed evolution of the rotation and $T_{e}$ profiles for the case in figure 7 are shown in figure 8 . Figure 8(a) shows that multiple LMs slow the rotation to near zero from the $q=2$ rational surface to the plasma edge. The central rotation profile inside the $q=2$ rational surface, however, is almost the same as that of single $2 / 1 \mathrm{EF}$ penetration in figure 5 . This is expected, as only the zero rotation point at $q=2$ determines the boundary of central rotation. After the excitation of multiple LMs, $T_{e}$ is flattened at each rational surface, forming a stair-like $T_{e}$ profile as shown in figure $8(\mathrm{~b})$. Then the $T_{e}$ profile decreases quickly and globally accompanying with the growth of LMs. At the end of the simulation, the central $T_{e}$ is decreased by more than $50 \%$. This is more of a change than in the single $2 / 1 \mathrm{LM}$ case (figure 5). Furthermore, the $T_{e}$ profile between the $q=2$ and $q=4$ rational surfaces is nearly flattened even in this case in which there is no island overlap (evidenced by the plot of the magnetic topology shown in figure $8(\mathrm{c})$ ). The detailed evolution of the $T_{e}$ profile in figure $8(\mathrm{~b})$ also reveals that the outer edge boundary $T_{e}$ of the $3 / 1$ locked island is determined by the $4 / 1$ island, and that of $2 / 1$ locked island $T_{e}$ is in turn determined by the $3 / 1$ island. This compounding effect is distinct from the rotation evolution discussed previously. This progressive effect on the $T_{e}$ profile is a unique characteristic of multiple LMs on heat transport, and it is responsible for the much stronger reduction in $T_{e}$.

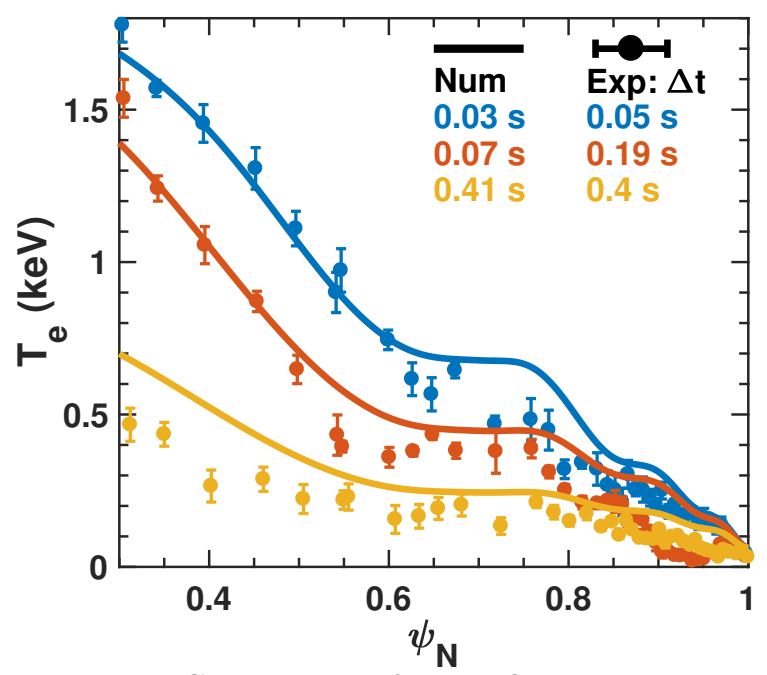

Figure 9. Comparison of $T_{e}$ profiles in the experiment and the modeling shown in figure 7 at $\Delta t=$ $0.05 \mathrm{~s}, 0.19 \mathrm{~s}, 0.4 \mathrm{~s}$ and $t=0.03 \mathrm{~s}, 0.07 \mathrm{~s}, 0.41 \mathrm{~s}$, respectively. Here, for experiment, $\Delta t=t-5.05 \mathrm{~s}$.

The experimental $T_{e}$ profiles are compared with numerical results in figure 9. Here, for the experimental profiles $\Delta t=t-5.05 \mathrm{~s}$. It is found that the modeled central $T_{e}$ profile is consistent with experiment inside the $q=2$ rational surface, but the edge $T_{e}$ evolves less and more slowly relative to the core $T_{e}$ in the modeling than in the experiment. The result is that the global experimental $T_{e}$ profile just before the TQ is lower than numerical $T_{e}$ in its saturated state $(t=0.41 \mathrm{~s})$. A possible reason is that the applied $3 / 1$ and $4 / 1 \mathrm{EF}$ amplitude are lower than they actually are in the experiment. The effect of the EF amplitude will be studied in the following. 


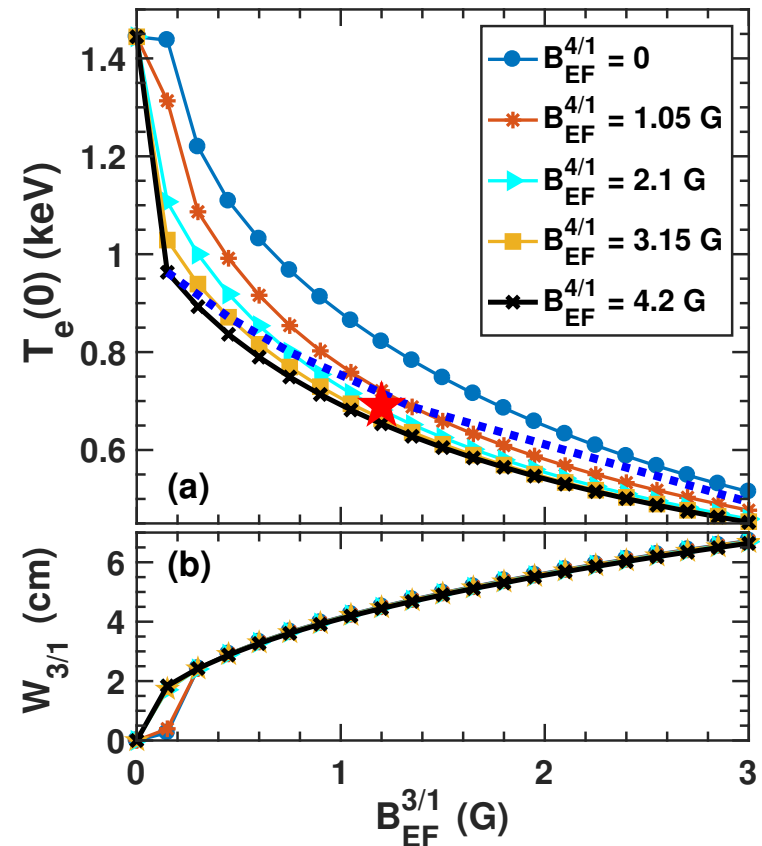

Figure 10. (a) Central electron temperature $T_{e}(0)$ and (b) $3 / 1$ island width $W_{3 / 1}$ are shown versus the amplitude of $3 / 1 \mathrm{EF}$ amplitude with different 4/1 EF amplitude. Island overlap happens for the results below the blue dotted curve, and the red five-pointed star presents the experimental EF amplitude.

The amplitude of the $3 / 1$ and $4 / 1$ EFs are scanned in the modeling, and the central $T_{e}(0)$ in the final saturated state is shown as a function of EF amplitude in figure 10. Here, the amplitude of the $2 / 1 \mathrm{EF}$ is fixed at the previous value of $2.3 \mathrm{G}$. It is found that for fixed amplitudes of $2 / 1$ and $4 / 1 \mathrm{EFs}$, stronger $3 / 1$ EF leads to a larger 3/1 island width and lower $T_{e}(0)$. A similar dependence of the $4 / 1$ island width on the $4 / 1 \mathrm{EF}$ amplitude is also found. The decrease in $T_{e}(0)$ per Gauss, however, becomes weaker with increasing $3 / 1$ or $4 / 1 \mathrm{EF}$ amplitude. In addition, edge stochasticity appears when island overlap happens between $3 / 1$ and $4 / 1$ locked islands or a large enough $4 / 1$ island approaches plasma edge. Here, the threshold boundary for edge stochasticity is indicated by the blue dotted curve in figure 10(a). No bifurcation or sharp change appears in $T_{e}(0)$ at the transition to stochastic fields, revealing the final $T_{e}$ depends purely on the island width and not the island overlap or stochasticity. The results in figures 11 and 12 will show, however, that the $T_{e}$ evolves to its final state much faster when there is island overlap.

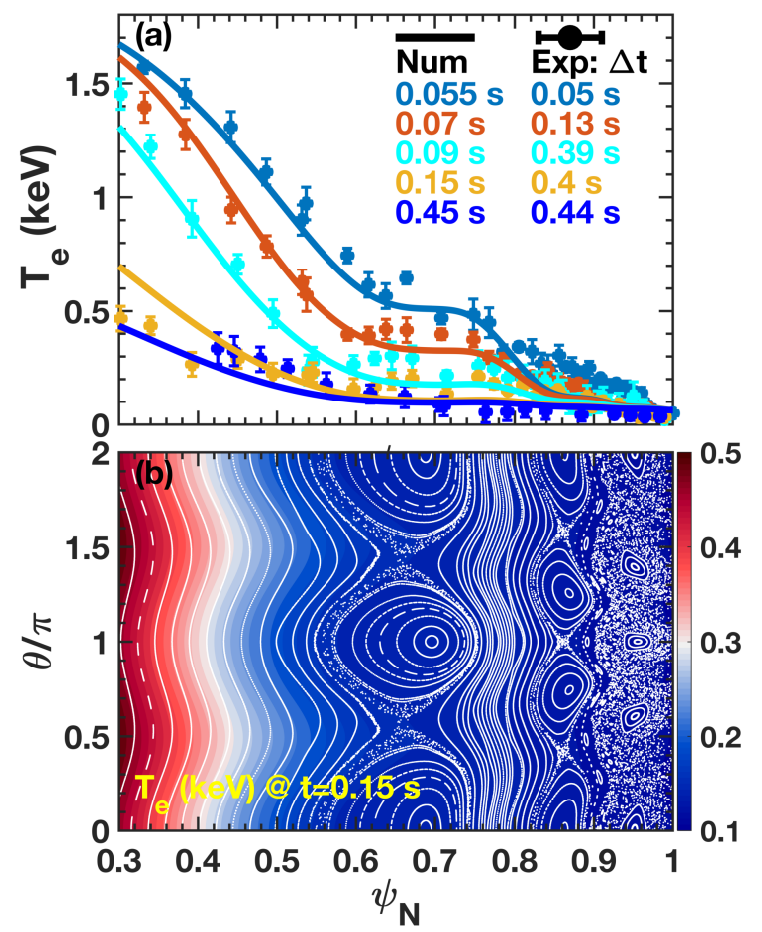

Figure 11. Modeling results using the experimental 2/1,3/1 and 4/1 EF amplitudes. (a) Comparison of $T_{e}$ profiles between experiment and modeling at $\Delta t=0.05 \mathrm{~s}, 0.13 \mathrm{~s}, 0.39 \mathrm{~s}, 0.4 \mathrm{~s}, 0.44 \mathrm{~s}$ and $t$ $=0.055 \mathrm{~s}, 0.07 \mathrm{~s}, 0.09 \mathrm{~s}, 0.15 \mathrm{~s}, 0.45 \mathrm{~s}$ respectively. (b) 2-D image of $T_{e}$ and Poincaré plot of the flux surfaces (white) at $t=0.15 \mathrm{~s}$ by modeling. Here, $\Delta t=t-5.05 s$.

As shown by the pentagram in figure 10, island overlap happens between $3 / 1$ and $4 / 1$ LMs with the experimental $2 / 1,3 / 1$ and $4 / 1$ EF amplitudes as shown in figure 11 . In this case, the evolution of $T_{e}$ profile is consistent with experiment. And the region from the 4/1 locked island to plasma edge becomes fully stochastic while the $3 / 1$ island chain still keeps a complete island structure as shown in figure 11(b). As a result, the peripheral plasma cools down fully as indicated by the $T_{e}$ profile, which approaches the edge temperature $T_{e}\left(\psi_{N}=1\right)$ from $q=2$ to the plasma edge.

When further increasing the amplitude of the $2 / 1,3 / 1$ and $4 / 1$ EFs to $5.5 \mathrm{G}, 2.1 \mathrm{G}$ and $3.1 \mathrm{G}$, the final $T_{e}$ profile is still reasonably consistent with experiment as shown in figure 12. In this case there is full stochasticity from the $3 / 1$ island region to the plasma edge, sec- 
ondary island structures with $m / n=5 / 2,3 / 2$, and harmonics inside the $2 / 1$ island. The final $T_{e}$ profile is even lower than the experimental profile after the TQ for both the cases in figures $11(t=0.45 \mathrm{~s})$ and $12(t=0.44 \mathrm{~s})$, indicating that multiple locked islands with overlap reproduce an effective TQ in the model.
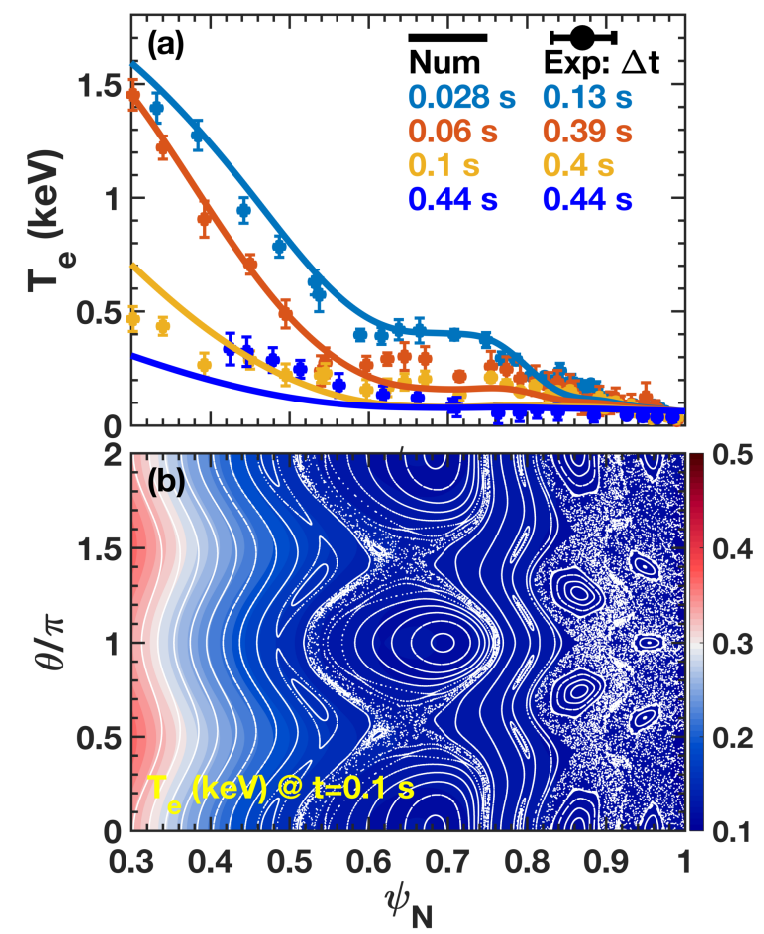

Figure 12. Stronger $2 / 1,3 / 1$ and $4 / 1 \mathrm{EFs}$ with amplitude of $5.5 \mathrm{G}, 2.1 \mathrm{G}$ and $3.1 \mathrm{G}$ lead to edge island overlap and secondary island structures. (a) Comparison of $T_{e}$ profiles between experiment and modeling at $\Delta t=0.13 \mathrm{~s}, 0.39 \mathrm{~s}, 0.4 \mathrm{~s}, 0.44 \mathrm{~s}$ and $t$ $=0.028 \mathrm{~s}, 0.06 \mathrm{~s}, 0.1 \mathrm{~s}, 0.44 \mathrm{~s}$, respectively. (b) 2-D profile of $T_{e}$ and Poincaré plot of the flux surfaces (white) at $t=0.1 \mathrm{~s}$ by modeling. Here, $\Delta t=t-5.05 s$.

\subsection{Influence of islands alignment at different phase}

In this section, the influence of islands alignment is studied by scanning the phase of each EF component separately.

The relative phase of modeled EF components can also affect the heat transport. Figure 13 shows the impact of scaning the phase of each component while the other components remain fixed. Here, the just-abovethreshold EF amplitudes shown in figure 7 are used. The central $T_{e}$ and island width are normalized by the corresponding values when the relative EF phases are all zero (as it was in all previous figures). When scanning the $2 / 1$ EF phase from 0 to $360^{\circ}$, the normalized central electron temperature $T_{e N}(0)$ changes sinusoidally with the magnitude of $3 \%$. At the same time, the island width of the $2 / 1$ and $4 / 1$ change little (less than $0.5 \%$ ) but the $3 / 1$ island width changes more with an magnitude of $4 \%$. Scanning the $3 / 1 \mathrm{EF}$ phase also causes periodical change in both $T_{e N}(0)$ and the island width. The magnitude is similar, but the oscillation period is twice of that of $2 / 1 \mathrm{EF}$ case. The results of scanning $4 / 1 \mathrm{EF}$ phase are essentially the same as scanning $2 / 1 \mathrm{EF}$ phase.

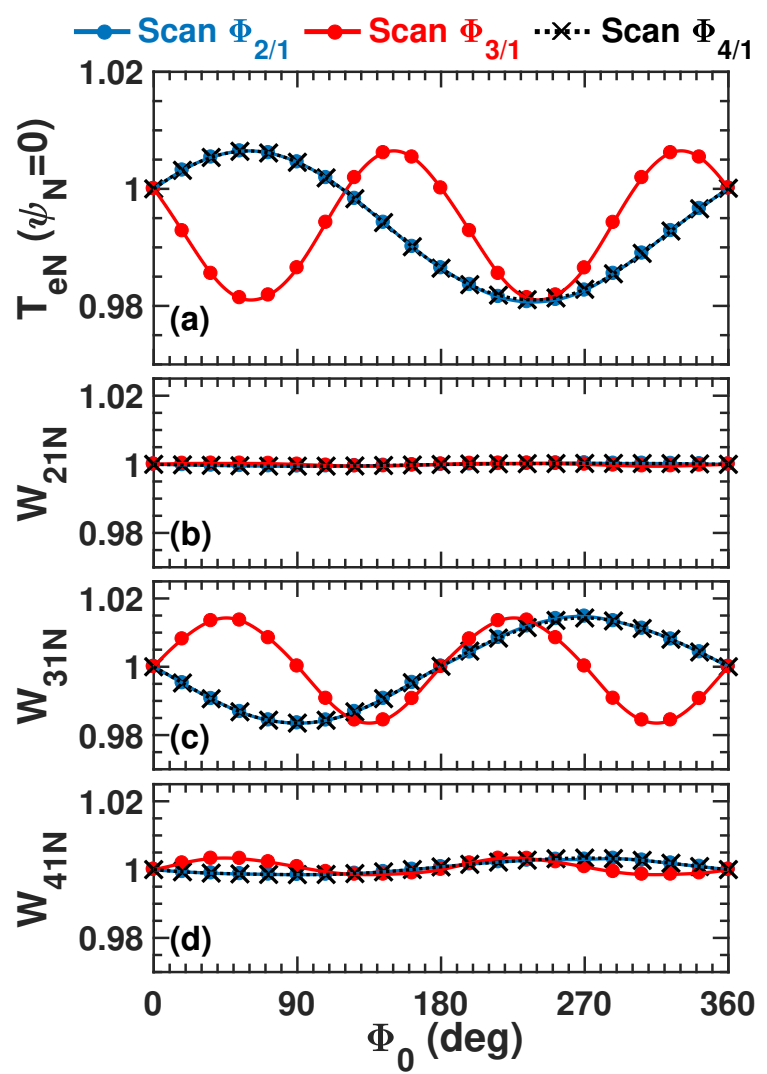

Figure 13. Influence of island phase on $T_{e}$ for none island overlap case. Normalized (a) central electron temperature $T_{e N}(0)$, (b) $2 / 1$ island width $W_{21 N}$, (c) $3 / 1$ island width $W_{31 N}$ and (d) $4 / 1$ island width $W_{41 N}$ versus EF phase. Here, applied EF amplitude are similar to that in figure 7, and the quantities presented here are normalized to the corresponding values when $\mathrm{EF}$ phase is zero.

Further studies show that, in the pres- 
ence of constant EF amplitude, the change in island width due to phase scanning is due to the influence of neighboring island chains, i.e. the magnetic island is stabilized by the magnetic perturbation contributed from neighboring island chains (or distorted flux surface as shown in figure 5) in certain phase alignment, while it is destabilized in other phase alignment. As a result, on the one hand, the widths of $2 / 1$ and $4 / 1$ island mainly depend on the magnetic perturbation of $3 / 1$ islands. On the other hand, the coexisting of $2 / 1$ and $4 / 1$ magnetic perturbations generates $m / n=6 / 2$ magnetic perturbation, which will further stabilize or destabilize $3 / 1$ island. That is why the $3 / 1$ island width is most sensitive to the phase of island chains, and also the results are the same when scanning the phase of $2 / 1$ and $4 / 1 \mathrm{EF}$.
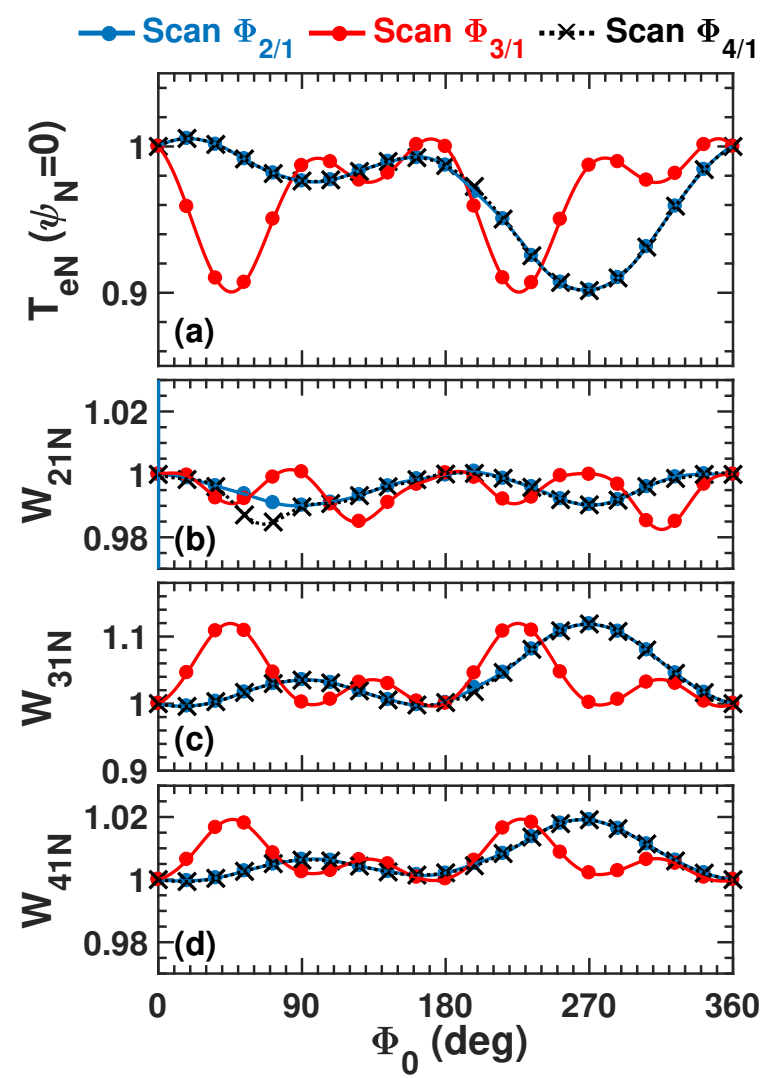

Figure 14. Influence of island phase on $T_{e}$ for island overlap case. Normalized (a) central electron temperature $T_{e N}(0)$, (b) $2 / 1$ island width $W_{21 N}$, (c) 3/1 island width $W_{31 N}$ and (d) $4 / 1$ island width $W_{41 N}$ versus EF phase. Here, applied EF amplitude are similar to that in figure 11, and the quantities presented here are normalized to the corresponding value when EF phase is zero.
The results of relative phase scans with the experimental EF component amplitudes are shown in figure 14. The change in both $T_{e N}(0)$ and island width are no longer sinusoidal function of EF phase. The magnitude of the change in $T_{e N}(0)$ is more than $10 \%$, and $W_{31 N}(14 \%)$ changes more than $W_{21 N}(2 \%)$ and $W_{41 N}(2 \%)$. The relative phase of experimental EFs between 2/1 (4/1) and 3/1 locates in the region of $100^{\circ}<\Phi_{0}<200^{\circ}$, indicating the temperature and saturated island width are similar to the case with the same EF phase. Detailed studies reveal that the non-sinusoidal dependence on the EF phase is due to the larger island widths as well as edge island overlap, resulting in the alignment of different island chains at specific phase.

Figures 13 and 14 indicate that the 3/1 island width is most sensitive to the phase of island chains (EF phase), and the change in $T_{e N}(0)$ is correlated with $W_{31 N}$. Namely, larger $W_{31 N}$ leads to lower $T_{e N}(0)$ and smaller $W_{31 N}$ leads to higher $T_{e N}(0)$.

\section{Discussion and summary}

Multiple helicity EF penetration and its effect on heat transport are investigated in this paper using resistive single fluid MHD equations with a cylindrical circular tokamak approximation. This cylindrical geometry is different from the DIII-D highly shaped plasma. As a result, on the one hand, this approximation neglects the modifications of plasma response expected from a toroidal plasma ideal MHD modes, which will modify tearing parity resonant fields at $q=2,3$ and 4 . On the other hand, the toroidal mode coupling effect is neglected under this approximation. This effect will generate magnetic perturbations with broad spectrum [23, 24], which in turn further advances the heat transport under multiple LMs. In the modeling, the central rotation profile is not near zero, however the global rotation is almost zero after mode locking or field penetration in DIII-D experiments [24], indicating there should be additional LMs, momentum transport or changes 
in the momentum sources inside the $q=2$ rational surface and their effect should be taken into account. Future work to use a full toroidal geometry would allow one to further take into account these effects and to give a more precise consistence with experiment. Nevertheless,even despite the above effects, the results in this paper reveal that the co-existence of multiple LMs deteriorate plasma thermal confinement much more than the sum of their isolated impacts would, and the observed $T_{e}$ profile in DIII-D experiment is qualitatively reproduced.

It is well known that an evolving $T_{e}$ profile can affect the plasma equilibrium and current decay [50]. On one hand, the decreased $T_{e}$ due to multiple LMs will increase the plasma resistivity and decrease the resistive decay time, which in turn changes the time scale for both LMs saturation and heat transport. On the other hand, the increased resistivity will cause redistribution of current density in the resistive time scale [28]. Besides, the plasma toroidal loop voltage increases due to the increased resistivity and results in higher Ohmic heating power (figure 2(b)). The increased Ohmic power would distribute more in the central plasma due to the peaked current density, leading to relatively higher central $T_{e}$ [38]. The above effects couple together in the experiment, but the multi-time scale physics is dificult to capture numerically. These effects have not been fully included in our model here, and may be a reason for the inconsistent time scales between experiment and modeling. However, these details do not affect the qualitatively understanding of heat transport under multiple LMs.

Stochasticity is thought to be a possible reason for the thermal collapse and TQ in LM disruptions $[17,18]$. This work shows that island overlap forms field stochasticity when the island widths are large enough and that it makes the $T_{e}$ evolve much faster as shown in figures 11 and 12. When a stochastic 4/1 island covers the plasma edge, the plasma outside the $q=3$ surface cools very rapidly. This kind of edge stochastic field degrades energy confinement, exhausts particles and shrinks the current density distribution quickly [51], resulting in loss of equilibrium torque balance. These results are consistent with the stochastic edge being largely responsible for the fast TQ preceding major disruptions. Furthermore, the avoidance of multiple LMs but not single helicity LM will be important to avoid disruption for future reactor devices.

In summary, in order to understand the heat transport with multiple LMs, this work studies $m / n=2 / 1,3 / 1$ and $4 / 1$ EF penetration and the effect of that penetration on heat transport using nonlinear reduced MHD equations. The conclusion of the work are:

(1) Multiple helicity EFs accelerate the occurrence of field penetration due to the braking EM torque at each rational surface slowing the global rotation profile.

(2) The coexistence of multiple LMs causes a reduction in $T_{e}$ of more than $50 \%$, which is much higher than that of a single $2 / 1 \mathrm{LM}(20 \%)$. The additive effect of multiple LMs effectively flattens the $T_{e}$ profile from $q$ $=2$ to the $q=4$ rational surface even without island overlap.

(3) The experimental post-TQ $T_{e}$ profile is reproduced when using the experimental EF amplitudes in the model. This produces island overlap between the $3 / 1$ and 4/1 LMs. Stronger EF amplitude leads wider stochastic region and lower $T_{e}$ profile, and even triggers secondary island structures.

(4) Scans of the relative phase between the EF components show that the $3 / 1$ island width is most sensitive to the relative phases, and the changed central $T_{e}(0)$ correlates with the $3 / 1$ island width.

\section{Acknowledgments}

The authors would like to thank E.J. Strait and R.J. Buttery for carefully reviewing the manuscript. The experimental target plasma described here was obtained in the DIII-D National User Facility operated by General Atomics in San Diego, CA. This work is supported by the US. Department of Energy un- 
der contract number DE-AC02-09CH11466, DE-FOA-0001386, DE-SC0015878 and DEFC02-04ER54698. DIII-D data shown in this paper can be obtained in digital format by following the links at https://fusion.gat com/global/D3D_DMP.

Disclaimer-This report was prepared as an account of work sponsored by an agency of the United States Government. Neither the United States Government nor any agency thereof, nor any of their employees, makes any warranty, express or implied, or assumes any legal liability or responsibility for the accuracy, completeness, or usefulness of any information, apparatus, product, or process disclosed, or represents that its use would not infringe privately owned rights. Reference herein to any specific commercial product, process, or service by trade name, trademark, manufacturer, or otherwise, does not necessarily constitute or imply its endorsement, recommendation, or favoring by the United States Government or any agency thereof. The views and opinions of authors expressed herein do not necessarily state or reflect those of the United States Government or any agency thereof.

\section{References}

[1] Schuller F. C. Plasma Phys. Control. Fusion 37 (1995) A135.

[2] ITER Physics Expert Group on Disruptions, Plasma Control, and MHD and ITER Physics Basis Editors Nucl. Fusion 39 (1999) 2251.

[3] Hender T. C. et al. Nucl. Fusion 47 (2007) S128.

[4] de Vries P. C. et al. Nucl. Fusion 49 (2009) 055011.

[5] de Vries P. C. et al. Nucl. Fusion 51 (2011) 053018 .

[6] Sweeney R. et al. Nucl. Fusion 57 (2017) 016019.

[7] Gerhardt S. P. et al. Nucl. Fusion 53 (2013) 043020.

[8] Nave M. F. F. et al. Nucl. Fusion 30 (1990) 2575.

[9] Hender T. C. et al. Nucl. Fusion 32 (1992) 2091.

[10] Fitzpatrick R. Nucl. Fusion 33 (1993) 1049.

[11] Buttery R. J. et al. Nucl. Fusion 39 (1999) 1827.
[12] Wesson J. A. et al. Nucl. Fusion 29 (1989) 641.

[13] Suttrop W. et al. Nucl. Fusion 37 (1997) 119.

[14] McGuire K. M. et al. Phys. Rev. Lett. 44 (1980) 1666.

[15] Sauthoff N. R. et al. Nucl. Fusion 18 (1978) 1445.

[16] Tsuji S. et al. Nucl. Fusion 25 (1985) 305.

[17] Choi M. J. et al. Nucl. Fusion 56 (2016) 066013.

[18] Sweeney R. et al. Nucl. Fusion 58 (2018) 056022 .

[19] Du X. D. et al. Measurements of the Key Role of Multiple, Small Locked Edge Islands in Triggering Tokamak Disruption Phys. Rev. Lett. (2018) in preparation.

[20] Scoville J. T. et al. Nucl. Fusion 43 (2003) 250.

[21] Paz-Soldan C. et al. Phys. Plasmas 21 (2014) 072503.

[22] Fitzpatrick R. et al. Nucl. Fusion 33 (1993) 1533.

[23] Park J.-k. et al. Phys. Plasmas 14 (2007) 052110.

[24] Tobias B. et al. Phys. Plasmas 23 (2016) 056107.

[25] Rechester A. B. et al. Phys. Rev. Lett. 40 (1978) 38.

[26] Turner M. F. et al. Nucl. Fusion 22 (1982) 1069.

[27] Bondeson A. Nucl. Fusion 26 (1986) 929.

[28] Taylor P. L. et al. Phys. Rev. Lett. 76 (1996) 916.

[29] Chang Z. et al. Nucl. Fusion 30 (1990) 219.

[30] Fitzpatrick R. Phys. Plasmas 2 (1995) 825.

[31] de Vries P. C. et al. Nucl. Fusion 56 (2016) 026007.

[32] Park J.-K. et al. Nucl. Fusion 51 (2011) 023003.

[33] Paz-Soldan C. et al. Nucl. Fusion 54 (2014) 073013.

[34] Haye R. J. L. et al. Nucl. Fusion 55 (2015) 023011.

[35] La Haye R. J. et al. Rev. Sci. Instrum. 62 (1991) 2146.

[36] Schaffer M. J. et al. Nucl. Fusion 48 (2008) 024004 . 
[37] Austin M. E. et al. Rev. Sci. Instrum. 74 [45] Günter S. et al. J. Comput.Phys. 209 (2005) (2003) 1457. 354.

[38] de Vries P. C. et al. Plasma Phys. Control. [46] Yu Q. Phys. Plasmas 13 (2006) 062310. Fusion 39 (1997) 439.

[47] Yu Q. et al. Nucl. Fusion 48 (2008) 024007.

[39] Hölzl M. et al. Nucl. Fusion 49 (2009) 115009.

[40] Yu Q. et al. Phys. Plasmas 10 (2003) 797.

[48] Chang Z. et al. Physics of Fluids B: Plasma Physics 4 (1992) 1167.

[41] Yu Q. et al. Phys. Plasmas 11 (2004) 1960. [49] Yu Q. et al. Phys. Plasmas 16 (2009) 042301.

[42] Yu Q. et al. Nucl. Fusion 48 (2008) 065004.

[43] Hu Q. et al. Nucl. Fusion 52 (2012) 083011.

[50] Kawakami S. et al. Phys. Plasmas 20 (2013) 112507.

[44] Hu Q. et al. Phys. Plasmas 20 (2013) 092502. [51] Ghendrih P. et al. Plasma Phys. Control. Fusion 38 (1996) 1653. 